\title{
OPEN Stress-induced differential gene expression in cardiac tissue
}

\author{
Ana Elisa T. S. de Carvalho ${ }^{\bowtie}$, Marco A. Cordeiro, Luana S. Rodrigues, Daniela Ortolani \& \\ Regina C. Spadari
}

The stress response is adaptive and aims to guarantee survival. However, the persistence of a stressor can culminate in pathology. Catecholamines released as part of the stress response over activate beta adrenoceptors ( $\beta$-AR) in the heart. Whether and how stress affects the expression of components of the intracellular environment in the heart is still, however, unknown. This paper used microarray to analyze the gene expression in the left ventricle wall of rats submitted to foot shock stress, treated or not treated with the selective $\beta_{2}$-AR antagonist ICI118,551 (ICI), compared to those of non-stressed rats also treated or not with $\mathrm{ICl}$, respectively. The main findings were that stress induces changes in gene expression in the heart and that $\beta_{2}$-AR plays a role in this process. The vast majority of genes disregulated by stress were exclusive for only one of the comparisons, indicating that, in the same stressful situation, the profile of gene expression in the heart is substantially different when the $\beta_{2}$-AR is active or when it is blocked. Stress induced alterations in the expression of such a large number of genes seems to be part of stress-induced adaptive mechanism.

The stress response is characterized by the activation of the sympathetic nervous system-adrenal medulla, which releases catecholamines, as well as the activation of the hypothalamic-pituitary-adrenal axis, which increases the secretion of glucocorticoids. Catecholamines and glucocorticoids, the hallmarks of the stress response, play fundamental roles as physiological regulators in an attempt to maintain homeostasis and adapt to a new condition. The persistence of the stressful situation may even culminate in increased susceptibility to certain diseases. High levels of catecholamines overstimulate adrenoceptors in the cell membrane of almost every organ, including the $\beta$-adrenoceptors $\left(\beta\right.$-AR) in the cardiomyocytes ${ }^{1}$.

In the human and rodent heart, the main $\beta$-AR subtype expressed is $\beta_{1}$-AR over $\beta_{2}$-AR in a proportion of $80: 20^{2-4}$. Both, $\beta_{1}$ - and $\beta_{2}$-AR couple to $\mathrm{G}$ stimulatory (Gs) protein, which stimulates adenylyl cyclase (AC) to convert ATP in $3^{\prime}-5^{\prime}$-cyclic adenosine monophosphate (cAMP), which then activates protein kinase A (PKA) ${ }^{1}$. Targets of PKA include L-type calcium channels, T troponin and phospholamban ${ }^{4}$. The phosphorylation of these proteins contributes an increase in the beating rate, development of tension, and the velocity of relaxation of the cardiomyocytes, thus increasing cardiac output. $\beta_{2}$-AR may also couple to G inhibitory (Gi) protein, with effects opposite to those of Gs stimulation. $\beta_{2}$-AR-Gi also activates the phosphatidylinositol 3-kinase (PI3K)-Akt signaling pathway, which controls the life and death of cardiomyocytes ${ }^{5}$.

Several studies have already shown the effects of stress in triggering alterations in the cardiovascular system $^{6-13}$. Such alterations can also trigger the onset of diseases, such as atherosclerosis, coronary diseases ${ }^{12,14}$, hypertension, and heart failure ${ }^{11,12}$. Our research group has invested substantial efforts in the investigation of the effects of stress on cardiac reactivity to $\beta$-AR stimulation in animal models of stress, mainly the foot shock stress $\operatorname{model}^{10,13}$. We have reported that isolated atria of rats submitted to foot shock stress show altered sensitivity to the chronotropic and inotropic effects of catecholamines, which is associated with a remodeling of the proportion of $\beta$-AR subtypes in the heart. Isolated atria were subsensitive to $\beta_{1}$-AR agonists and supersensitive to both $\beta_{2}$-AR selective agonists and isoprenaline, a non-selective $\beta$-AR agonist. These effects of stress were cancelled in the presence of selective $\beta_{2}$-AR antagonists or non-selective $\beta$-AR antagonists, suggesting an increase in the $\beta_{2}$-AR subtype in the cardiac tissue ${ }^{10,13,15,16}$. A Western blot approach confirmed the higher expression of $\beta_{2}$-AR accompanied by a low expression of $\beta_{1}$-AR in the atrium and ventricle of rats submitted to foot shock stress when compared to control non-stressed rats ${ }^{13}$. A reduced cardiac $\beta_{1} / \beta_{2}$-AR ratio is also involved in other circumstances, such as aging and heart failure. It is considered adaptive since it protects the heart from the cardiotoxic effects of persistent $\beta_{1}$-AR overstimulation ${ }^{1,5,17,18}$

In contrast to the recognized cardiotoxic effect of persistent $\beta_{1}$-AR stimulation, the possible cardioprotective role of prolonged $\beta_{2}$-AR activation is still controversial. After the receptor couples with Gi, the Gia subunit

Laboratory of Stress Biology, Department of Biosciences, Institute of Health and Society, Campus Baixada Santista, Federal University of São Paulo (UNIFESP), Rua Silva Jardim,136, sala 310, Santos, São Paulo 11020-015, Brazil.

『email: aetscarvalho@unifesp.br; regina.spadari@unifesp.br 


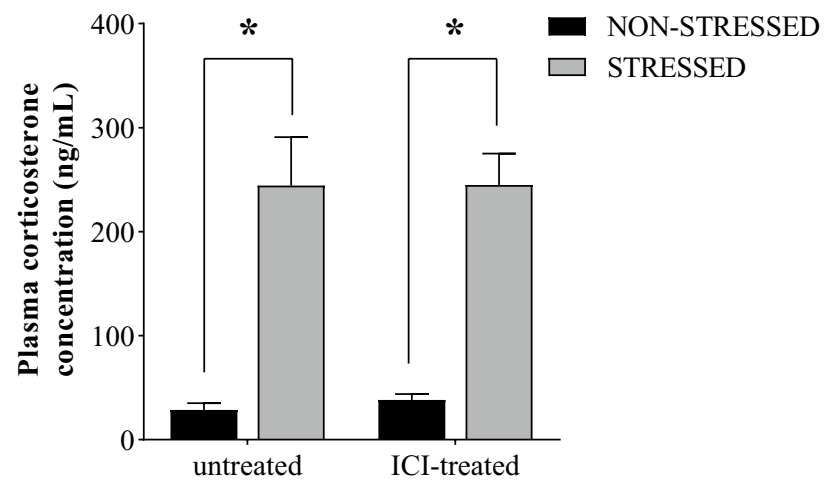

Figure 1. Plasma concentration of corticosterone in untreated non-stressed control rats, untreated rats submitted to stress, non-stressed rats treated with ICI118,551, and rats both treated with ICI118,551 and submitted to stress. The bars indicate mean \pm S.E.M. of 6 rats/group. ${ }^{*} \mathrm{p} \leq 0.05$ compared to control, two-way ANOVA plus Tukey test.

inhibits $\mathrm{AC}$, attenuating the signal mediated by $\beta_{1}-\mathrm{AR}$, while the Gi $\beta \gamma$ activates several intracellular signals, including PI3K-Akt ${ }^{19,20}$. Despite the beneficial effect on the viability of cardiac cells, $\beta_{2}$-AR stimulation compromises contractility. Recently, we have demonstrated that the activity of PI3K-Akt is reduced in the heart of foot shock stressed rats even 5 days after the last stress session ${ }^{21}$. The persistence of this effect indicates the relevance of the intracellular processes triggered by stress in the heart. Moreover, the increase in $\beta_{2}$-AR participation in the cardiac stress response and heart failure suggests an important role for $\beta_{2}$-AR signaling in cardiac events. However, the complex biological processes contributing to the cardiac response to stress are not yet fully understood.

To contribute to our understanding of the influence of stress on cardiac physiology, we have proposed an evaluation of the gene expression profile in the cardiac tissue of rats submitted to foot shock stress. We have also compared the gene expression profile in the presence of stress-induced upregulation of $\beta_{2}-A R$ and in the absence of $\beta_{2}$-AR signaling due to the pharmacological blockade of the receptor. Our results provide insight into the role played by $\beta_{2}$-AR in the differential regulation of gene expression during stress.

\section{Results}

The corticosterone plasma level was higher in the rats submitted to foot shock stress than in non-stressed rats. The $\beta_{2}$-AR blockade with ICI118,551 did not interfere with this endocrine stress response (Fig. 1).

$\beta_{2}$-AR drives gene expression in the heart post stress. In order to identify the genes of which expression was altered in the heart of rats submitted to stress, samples were arranged for microarray so that untreated rats with and without stress were compared. Since $\beta_{2}$-AR is upregulated in the heart of stressed rats ${ }^{13,21}$, its influence on target genes expression under stress was also evaluated with ICI treatment (with or without stress).

In microarray analysis, 265 genes were found to be significantly disregulated in non-ICI treated rats, with 208 upregulated and 57 downregulated under stress. $\beta_{2}$-AR blockade resulted in the significant disregulation of 114 genes under stress, with 90 being upregulated and 14 downregulated. The functional analysis of the genes with differential expression was performed using IPA software. Interestingly, many non-annotated genes were disregulated in both comparisons, but mainly those in the comparison of the untreated groups (Table 1, Supplementary Tables S1, S2).

The genes with the greatest differential expressions are listed in Table 1. In the comparison of untreated rats, the genes RGS1 (regulator of G protein signaling), PDLIM3 (PDZ and LIM domain 3), EGR1 (early growth response 1), CREM (cAMP) responsive element modulator) and IL6R (interleukin 6 receptor) underwent the greatest upregulation in the presence of stress, while MYCN (MYCN proto-oncogene), TLR3 (toll like receptor 3 ) and MYBPC2 (myosin binding protein C2) were downregulated.

For the comparison of the ICI-treated groups (with versus without stress), the greatest upregulation was found for the genes coding heat shock proteins (HSPA1A/HSPA1B and HSP90AA1), insulin receptor substrate 2 (IRS2) and ATF3 (activating transcription factor 3), whereas SEZ6L (seizure related 6 homolog like), CCL2 (C-C motif chemokine ligand 2) and APLNR (apelin receptor) underwent downregulation (Table 1).

There was minimal overlap in the genes disregulated by stress in the two comparisons, as can be seen in the Venn diagram (Fig. 2). Three genes proved to be upregulated under stress, independent of the presence of the $\beta_{2}$-AR: cAMP responsive element modulator (CREM), ERBB receptor feedback inhibitor 1 (ERRFI1), and heat shock protein 90 alpha family class A member (HSP90AA1). The vast majority of disregulated genes were specific for one of the conditions, with 166 annotated genes being disregulated in cardiac tissue when $\beta_{2}$-AR was active (ICI-untreated), and 76 when $\beta_{2}$-AR was blocked (ICI-treated).

Stress induces alterations in the expression of genes related to inflammatory response, cell cycle and proliferation. An analysis of the disregulated genes suggests that certain categories of diseases and functions may be triggered or altered in the cardiac tissue of rats submitted to stress (Supplementary Table S3). Figure 3 shows some of these categories of diseases and altered function. In the comparison of the 


\begin{tabular}{|c|c|c|c|c|}
\hline & \multicolumn{2}{|l|}{ Untreated } & \multicolumn{2}{|l|}{ ICI-treated } \\
\hline & Gene & Log ratio & Gene & Log ratio \\
\hline & LOC102S56673* & 3.327 & HSPA1A/HSPA1B & 3.867 \\
\hline & LOC102551486 & 2.572 & APOLD1 & 2.195 \\
\hline & RGS1 & 2.489 & IRS2 & 2.132 \\
\hline & PDLIM3 & 2.168 & Sik1 & 2.041 \\
\hline \multirow{10}{*}{ UP } & LOC103691S66 $^{*}$ & 2.12 & PER1 & 2.016 \\
\hline & EGR1 & 2.087 & TRIM21 & 2.029 \\
\hline & LOC103693175* & 1.966 & USP2 & 1.907 \\
\hline & CREM & 1.933 & HSP90AA1 & 1.836 \\
\hline & IL6R & 1.923 & STC1 & 1.828 \\
\hline & LOC100909409* & 1.909 & ATF3 & 1.814 \\
\hline & MYCN & -2.039 & SEZ6L & -2.84 \\
\hline & C2CD4A & -1.634 & CCL2 & -2.171 \\
\hline & CDKN2C & -1.554 & SGCB & -1.904 \\
\hline & GSTA5 & -1.534 & Histlhlb & -1.684 \\
\hline \multirow{6}{*}{ DOWN } & PTGDS & -1.458 & PM20D2 & -1.341 \\
\hline & RRAD & -1.41 & KIF22 & -1.319 \\
\hline & TLR3 & -1.402 & APLNR & -1.31 \\
\hline & MYBPC2 & -1.399 & LOC102548611* & -1.277 \\
\hline & TTC30B & -1.366 & ARNTL & -1.238 \\
\hline & IFi47 & -1.307 & ART5 & -1.146 \\
\hline
\end{tabular}

Table 1. Most disregulated genes identified in the heart by the Ingenuity Pathway Analysis based on the comparison of stressed with non-stressed untreated rats (4 rats/group), and those stressed and unstressed treated with ICI118,551 (4 rats/group). The disregulated genes were ranked according to experimental $\log$ ratio. Thresholds were based on extent of fold change $(\geq 2)$ and $p$ value $\leq 0.05$. The lists with additional information are available in Supplementary Tables S1 and S2. ${ }^{\star}$ Non-annotated genes.

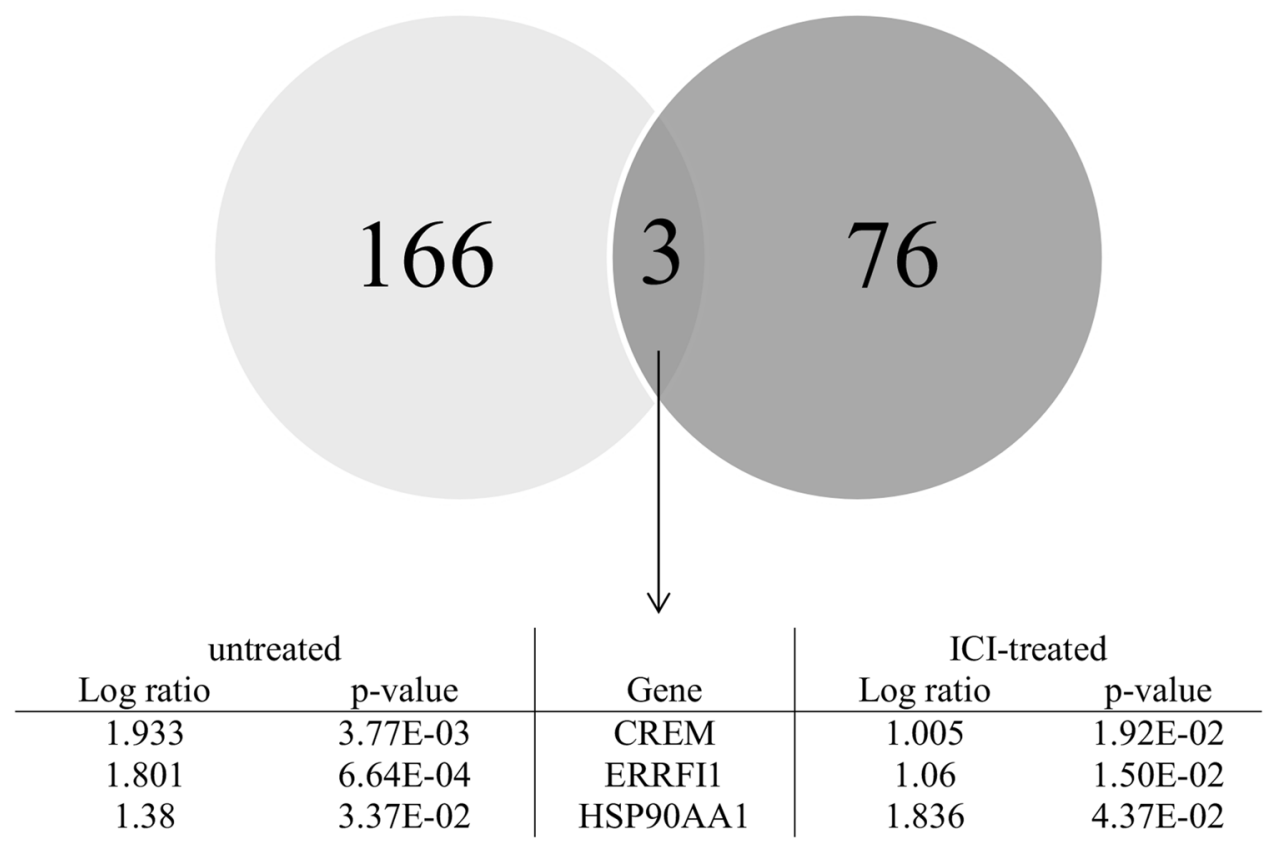

Figure 2. Venn diagram showing the number of disregulated genes by stress based on the comparison of stressed with non-stressed untreated rats (light gray; 4 rats/group), and those stressed and unstressed treated with ICI118,551 (dark gray; 4 rats/group). Only three genes were affected in the two comparisons. The list of affected genes includes name, location, type, $p$ value, and log ratio. 


\section{untreated}

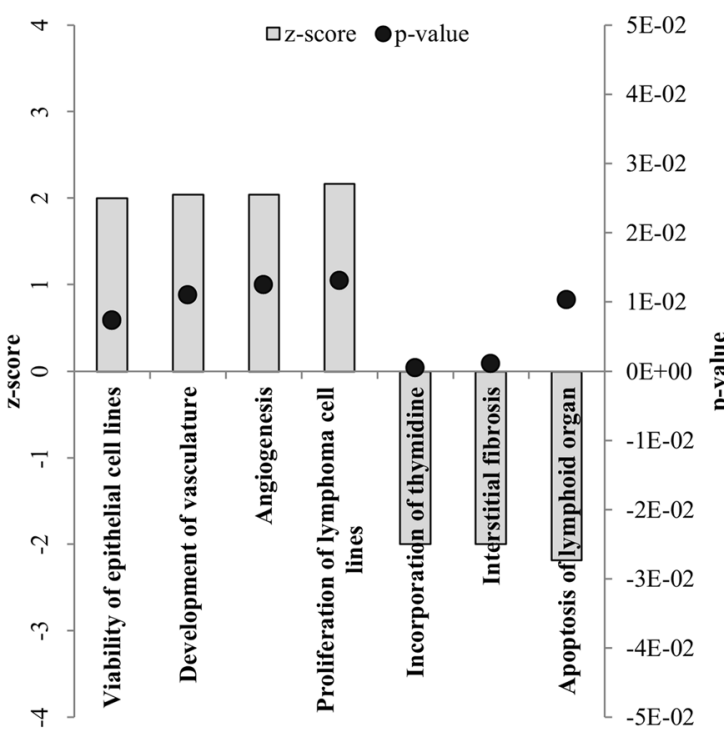

ICI-treated

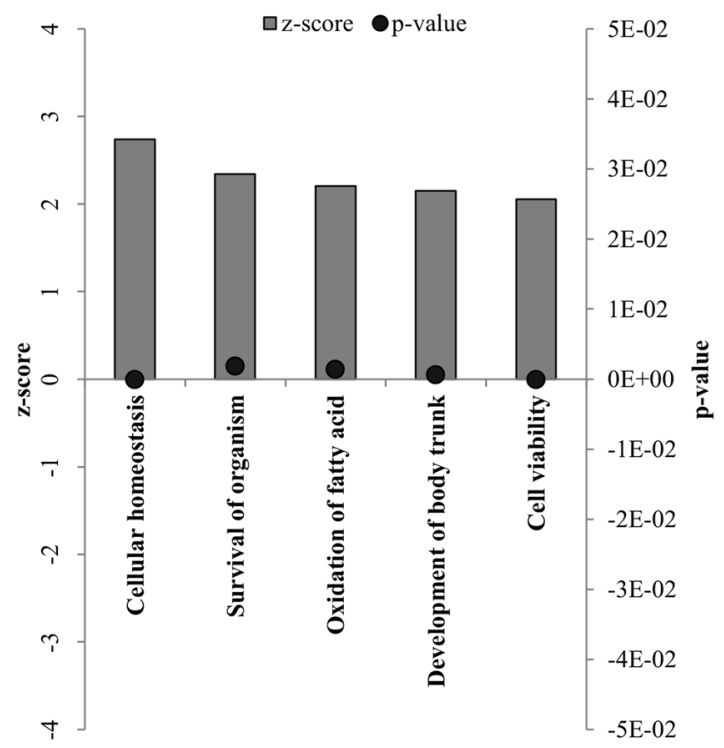

Figure 3. Diseases and functions identified in the heart by the Ingenuity Pathway Analysis based on the comparison of stressed with non-stressed untreated rats (4 rats/group), and those stressed and unstressed treated with ICI118,551 (4 rats/group). Bars indicate $z$-score, and black dots indicate the p value for each category. The thresholds were a $\mathrm{z}$ score of $\geq 2$ or $\leq-2$ and $\mathrm{p}$ value of $\leq 0.05$. A list with additional information is available in Supplementary Table S3.

\begin{tabular}{|l|l|}
\hline Untreated & ICI-treated \\
\hline Heme biosynthesis II & Glucocorticoid receptor signaling \\
\hline Eicosanoid signaling & IL17A signaling in fibroblasts \\
\hline Mitotic roles of polo-like kinase & IL17 signaling \\
\hline Cyclins and cell cycle regulation & Circadian rhythm signaling \\
\hline S-adenosyl-L-methionine biosynthesis & Protein ubiquitination pathway \\
\hline Cell cycle regulation by BTG family proteins & Acute phase response signaling \\
\hline Heme biosynthesis from uroporphyrinogen III I & Apelin endothelial signaling pathway \\
\hline Spermine and Spermidine degradation I & IL6 signaling \\
\hline Tetrapyrrole biosynthesis II & PI3K signaling in B lymphocytes \\
\hline \multirow{3}{*}{ Telomerase signaling } & CD27 signaling in lymphocytes \\
\cline { 2 - 2 } & CD40 signaling \\
\cline { 2 - 2 } & IL10 signaling \\
\cline { 2 - 2 } & cAMP mediated signaling \\
\hline
\end{tabular}

Table 2. Canonical pathways identified in the heart by the Ingenuity Pathway Analysis based on the comparison of stressed with non-stressed untreated rats (4 rats/group), and those stressed and unstressed treated with ICI118,551 (4 rats/group). Bold shading indicates prediction of activation and italics indicates prediction of inhibition. A list with additional information is available in Supplementary Table S4.

non-ICI treated groups, stress enhanced the expression of genes encoding by proteins related to angiogenesis and development of vasculature, epithelial cell viability, and proliferation of lymphoma cells, while reducing that of genes encoding proteins related to incorporation of thymidine, interstitial fibrosis and apoptosis of lymphoid organs. In ICI-treated groups, the presence of stress increased the expression of genes associated with homeostasis, survival, and the oxidation of fatty acids (Fig. 3).

The analysis of canonical pathways allows visualization of the signaling pathway in which the significantly disregulated genes are inserted, as well as their functional relevance. In the non-treated groups' comparison, no canonical pathway was activated, although pathways related to the inflammatory response, regulation of cell cycle and proliferation were listed (Table 2), with the most frequently affected genes in these pathways being ALAD (aminolevulinate dehydratase), CPOX (coproporphyrinogen oxidase), CDK1 (cyclin dependent kinase 1), HSP90AA1 (heat shock protein 90 alpha 1) and HDAC8 (histone deacetylase 8) (Supplementary Table S4). 


\begin{tabular}{|c|c|c|c|c|}
\hline Upstream regulator & Type & Predicted state & z score & p value \\
\hline \multicolumn{5}{|l|}{ Untreated } \\
\hline TNF & Cytokine & Activated & 3.088 & $7.32 \mathrm{E}-07$ \\
\hline IL1B & Cytokine & Activated & 2.967 & $8.07 \mathrm{E}-10$ \\
\hline $\mathrm{F} 2$ & Peptidase & Activated & 2.757 & $4.20 \mathrm{E}-04$ \\
\hline CG & Complex & Activated & 2.701 & $4.00 \mathrm{E}-05$ \\
\hline $\mathrm{Ca}^{+2}$ & Chemical-endogenous & Activated & 2.586 & $1.80 \mathrm{E}-03$ \\
\hline GPER1 & G-protein coupled receptor & Activated & 2.425 & $8.28 \mathrm{E}-07$ \\
\hline MAPK1 & Kinase & Activated & 2.414 & $2.60 \mathrm{E}-03$ \\
\hline MAPK8 & Kinase & Activated & 2.391 & $9.69 \mathrm{E}-04$ \\
\hline SMARCA4 & Transcription factor & Activated & 2.213 & $4.45 \mathrm{E}-02$ \\
\hline ACOX1 & Enzyme & Inhibited & -2.0 & $1.87 \mathrm{E}-02$ \\
\hline \multicolumn{5}{|l|}{ ICI-treated } \\
\hline CREB1 & Transcription factor & Activated & 3.634 & $1.40 \mathrm{E}-12$ \\
\hline Insulin & Group & Activated & 3.035 & $1.15 \mathrm{E}-15$ \\
\hline NUPR1 & Transcription factor & Activated & 3.0 & $6.56 \mathrm{E}-05$ \\
\hline PGR & Ligand-dependent nuclear & Activated & 2.96 & $6.53 \mathrm{E}-09$ \\
\hline GPER1 & G-protein coupled receptor & Activated & 2.646 & $3.89 \mathrm{E}-10$ \\
\hline HIF1A & Transcription factor & Activated & 2.574 & $7.44 \mathrm{E}-04$ \\
\hline Norepinephrine & Chemical-endogenous & Activated & 2.553 & $1.43 \mathrm{E}-12$ \\
\hline Hydrogen peroxide & Chemical-endogenous & Activated & 2.528 & $8.19 \mathrm{E}-10$ \\
\hline EGE & Growth factor & Activated & 2.459 & $2.17 \mathrm{E}-08$ \\
\hline NKX2-3 & Transcription factor & Inhibited & -2.0 & $6.88 \mathrm{E}-03$ \\
\hline
\end{tabular}

Table 3. Most altered endogenous upstream regulators identified in the heart by the Ingenuity Pathway Analysis based on the comparison of stressed with non-stressed untreated rats (4 rats/group), and those stressed and unstressed treated with ICI118,551 (4 rats/group). The thresholds were $z$ score $(\geq 2$ or $\leq-2)$ and $p$ value $(\leq 0.05)$. The list with additional information is available in Supplementary Table S5.

Among the canonical pathways activated in the comparison of stressed and unstressed ICI-treated rats, the acute phase response signaling and IL6 signaling were positively regulated, while cAMP mediated signaling was negatively regulated (Table 2). Other pathways were listed including those associated with the Th17 inflammatory response, the regulation of the immune response, glucocorticoid and mineralocorticoid signaling, and cAMP signaling. Interestingly, most of pathways shared the same set of disregulated genes, including JUN (JUN protooncogene), MAP2K3 (mitogen-activated protein kinase 3), NFKBIA (NFkB inhibitor alpha), CEBPB (CCAAT enhancer binding protein beta), HSP90AA1 (heat shock protein 90 alpha 1), HSPA1A/HSPA1B (heat shock protein family A -Hsp70-members 1A and 1B), and DUSP1 (dual specificity phosphatase 1) (Supplementary Table S4).

Upstream molecules triggering gene expression changes. The IPA analysis of upstream regulators uses a knowledge data base to predict the upstream molecules that could be triggering the experimentally observed gene expression changes. This analysis suggested that the extra-cardiac environment was strikingly different in rats submitted to stress whether or not they had been treated with the $\beta_{2}$-AR blocker (Table 3, Supplementary Table S5). Table 3 shows the most altered upstream molecules. GPER1 (G protein-coupled estrogen receptor 1) was predicted to be an upstream regulator in the presence of stress, independent of ICI-treatment. It leads to AC activation, thus increasing cAMP levels. Very few upstream inhibitory regulators were identified (Table 3). The transcription factor CREB1 (cAMP responsive element binding protein 1) was the most altered upstream regulator for the comparison of ICI-treated groups (Table 3) but it was also present in the comparison of the untreated groups (Supplementary Table S5). CREB1 is the transcription factor that binds CREM as a response to an increase in cAMP levels. Among the upstream regulators with specific relevance in the comparison of non-ICI treated groups were pro-inflammatory cytokines, IL1B (interleukin 1 beta) and TNF (tumor necrosis factor), as well as calcium (Table 3), while the specific upstream regulators in the comparison of ICItreated groups were insulin and norepinephrine (Table 3).

\section{Discussion}

The data presented here were obtained by microarray technology that provided an overview of the level of mRNA in the heart of rats submitted to stress, treated or not treated with a $\beta_{2}$-AR antagonist. It was assumed that the level of mRNA reflects gene expression even though it may suffer post translational modifications, a process that might also be altered by stress, as it was previously reported ${ }^{22}$.

It has been shown that stress induces changes in gene expression and that $\beta_{2}$-AR modulates those changes in the heart. The vast majority of genes with expression disregulated by stress were different when $\beta_{2}$-AR was upregulated and when it was blocked by the ICI treatment; only three genes were disregulated in both cases. 
This indicates that, given the same stressful situation, the profile of gene expression in the heart is substantially different when $\beta_{2}$-AR is active or when it is blocked. The three genes with expression independent of $\beta_{2}$-AR were CREM, which is related to the cAMP signaling pathway, HSP90AA1, which encodes HSP90, the glucocorticoid receptor chaperone, and ERRFI1, which encodes the feedback inhibitor of the epidermal growth factor receptor, which attenuates the PI3K-Akt signaling pathway.

Effect of stress on genes related to cAMP signaling pathway. $\quad \beta$-AR activation by catecholamines is the main signal for cAMP generation in cardiac myocytes. The cAMP-dependent transcription factor, CREM, expressed in the myocardium, is involved in the regulation of the expression of various components of the cAMP signaling pathway ${ }^{23}$. It has been described as essential for normal cardiac function ${ }^{24,25}$ and required for the $\beta_{1}$-AR response to overstimulation ${ }^{25}$. CREM upregulation in the heart of stressed rats probably contributes for the efficacy of $\beta_{1}$-AR (with or without the participation of $\beta_{2}$-AR) signaling in the context of overstimulation. Moreover, many other genes involved in that same signaling pathway had their expression disregulated under stress, whether or not treated with ICI118,551. RGS1 and RGS16 were upregulated in the untreated groups' comparison. The RGS family acts as a negative regulator of $\mathrm{G}$ protein signaling. By controlling heterotrimeric $\mathrm{G}$ proteins they may regulate myocardial hypertrophy and contractility ${ }^{26}$. RGS1 has been related to the control of inflammation and the immune response $e^{27,28}$. The stress-induced alteration in the expression of these genes depends on an active $\beta_{2}$-AR, since it is unaltered in the group treated with ICI118,551. On the other hand, CREB1 and GPER1 belong to the group of molecules predicted to be upstream regulators activated by stress, independent of $\beta_{2}$-AR activation. CREB1, a transcription factor responsive to cAMP, leads to effects similar to CREM ${ }^{24}$, while GPER1 belongs to the G-protein coupled receptor family that binds estrogen. This receptor activates both the adenylate cyclase-cAMP/PKA signaling pathway ${ }^{29,30}$ and the PI3K-Akt-mTOR signaling pathway ${ }^{31}$. These two signaling pathways are related to cardioprotection and cell survival ${ }^{20}$.

The APLNR gene, also called the APJ receptor gene, encodes the G-protein coupled receptor for apelin, a protein expressed in the cardiovascular system which promotes a positive inotropic effect on the heart as well as angiogenesis and blood vessel relaxation ${ }^{32,33}$. Apelin not only increases inotropy, but also decreases left ventricular pre- and afterload due to its pronounced vasodilation effect ${ }^{34}$. The inotropic action of apelin is the result of an increase in the availability of intracellular calcium ${ }^{35}$. It also induces cAMP synthesis, as well as activating the PI3K-Akt signaling pathway ${ }^{33}$. The concentration of apelin is reduced in the failing heart ${ }^{36}$ and the contractile function is impaired in cardiomyocytes with knockout for APLNR ${ }^{37}$. Apelin expression was downregulated under stress when no ICI118,551 treatment was given, although the expression of its receptor (APLNR) was reduced when the $\beta_{2}$-AR was blocked.

Therefore, in the heart of untreated stressed rats, the downregulation of $\beta_{1}$-AR and apelin seems to be counterbalanced by the upregulation of $\beta_{2}$-AR and the action of CREM. Hence, although the proportion of $\beta$-AR subtypes is altered, there is no difference in cAMP formation by left atrial membranes of control and foot shock stressed rats stimulated by non-selective agonists ${ }^{13}$. However, when the membranes are stimulated in the presence of the $\beta_{2}$-AR antagonist (ICI118,551), the amount of cAMP synthetized by the atrial membranes of stressed rats is lower than that of unstressed ones ${ }^{13}$. This is probably because the increase in the expression of CREM is not sufficient to sustain the synthesis of cAMP, since $\beta_{1}$-AR is downregulated, as has been reported elsewhere ${ }^{13}$.

The present data thus confirm that when stress is applied under conditions of $\beta_{2}$-AR blockade, the canonical pathway of cAMP signaling is negatively regulated (see Table 2), due largely, but not only, to $\beta_{1}$-AR and APLNR downregulation. The increasing cAMP level due to $\beta_{1}$-AR and $\beta_{2}$-AR activation culminates in an increased rate of beating and force developed by isolated left atrium ${ }^{13}$. The calcium transient plays a central role in this process ${ }^{38}$. Indeed, calcium was predicted to be an upstream regulator in the presence of higher $\beta_{2}$-AR expression. Although extremely important for the proper functioning of cardiac cells, excessive calcium leads to disorders such as arrhythmia, hypertrophy, and cell death ${ }^{38}$. Therefore, the stress induced modulation of positive and negative influences on the expression of molecules related to cAMP and the calcium signaling pathways in the heart adds complexity, as well as more possibility for control of the cardiac function and structure, with $\beta_{2}$-AR apparently playing an essential role in the process.

Effect of stress on glucocorticoids signaling. Glucocorticoids (GC) and catecholamines are known as the stress hormones. The glucocorticoid receptor (GR) is located in the cytoplasm of target cells, bound to a chaperone complex and immunophilins that provide structural stability and function ${ }^{39}$. Upon the binding of the GC, the GR undergoes a conformational change that causes the release of the chaperones and dimerization of the complexes GC-GR. Then, the GC-GR dimers translocate to the nucleus, where they start its genomic signaling ${ }^{40,41}$. Increased corticosterone plasma levels led to an increase in GR-mediated genomic signaling and the modulation of gene expression.

The heat shock proteins (HSP) 90 and 70, as well as the proto-oncogene tyrosine-protein kinase (Src), released from the GR complex, also influence cell signaling ${ }^{41}$ by regulating protein folding, proteostasis and intracellular signal transduction. HSP90 and HSP70 are inducible isoforms that present incremented expression after myocardium injury, oxidative stress, and hypoxia. HSP90aa1, the mRNA for HSP90, was upregulated under stress, both with and without $\beta_{2}$-AR participation, while HSPa1a, the mRNA for HSP70, was upregulated by stress only under $\beta_{2}$-AR blockade. Upregulation of HSP90 and HSP70 has been linked to cardioprotection against injury ${ }^{39,42,43}$. The in vitro overexpression of HSP90aal in cardiomyocytes attenuates apoptosis by increasing Bcl-2 expression ${ }^{43}$. HSP90 is also associated with the survival pathway of PI3K-Akt. The association of HSP90 with Akt leads to phosphorylation and the activation of endothelial nitric oxide synthase ${ }^{39,42}$. Under stress, in the presence of $\beta_{2}$-AR upregulation, the reduction of fibrosis and apoptosis that was indicated by disease and function categorization, suggests that the modulation of the expression of genes is part of the adaptive mechanisms to stress. 


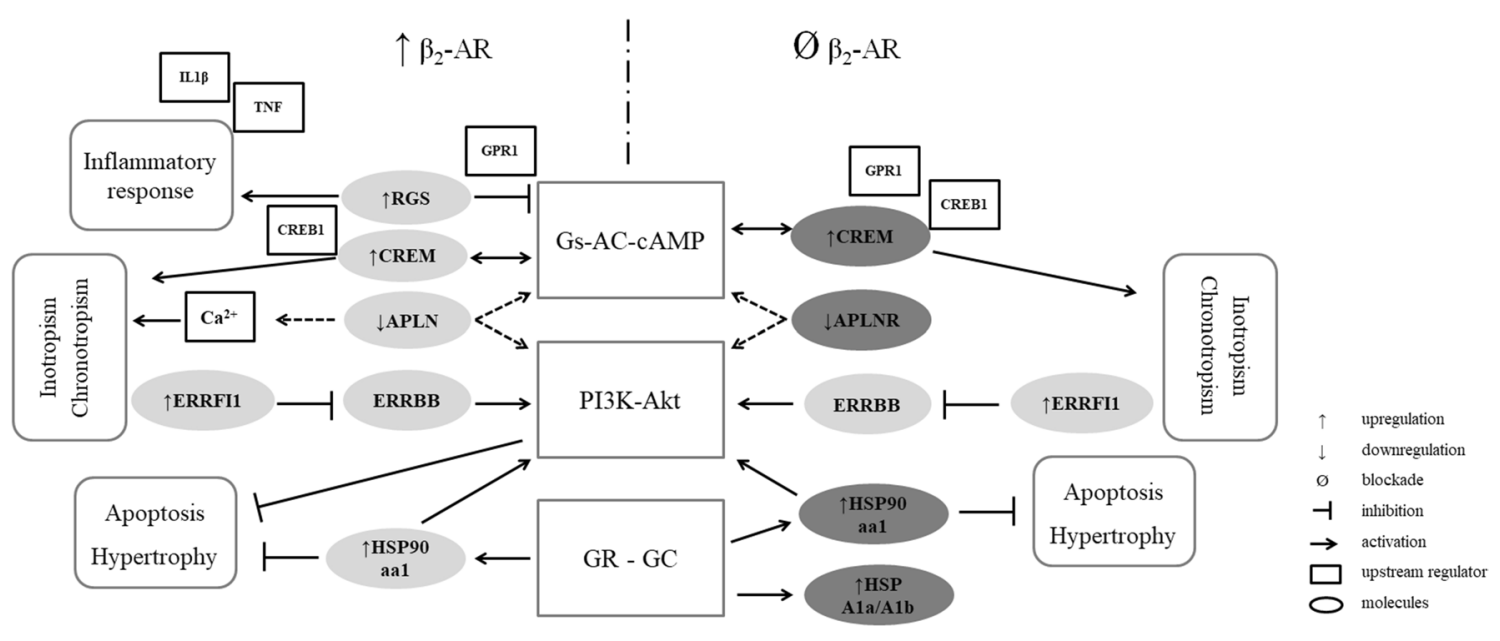

Figure 4. Schematic representation of the microarray analysis of the effect of stress on the expression of most disregulated genes in the ventricle of rats with $\beta_{2}$-adrenoceptors $\left(\beta_{2}\right.$-AR) upregulated (unstressed and stressed untreated rats comparison; left side) and those with the $\beta_{2}$-AR antagonized by ICI118,551 (comparison of unstressed and stressed ICI-treated rats; right side). The signaling pathways related to these genes are listed in the center of the figure, and the putative effect on cardiac function and structure are listed in the respective side of each condition.

The activation of GR signaling is also related to the induction of the expression of the gene ERRFI1. This gene encodes a feedback inhibitor of the epidermal growth factor receptor (EGFR) what reduces cardiac hypertrophy ${ }^{44,45}$. The results presented here have demonstrated that in rats submitted to stress as compared to non-stressed rats, the circulating levels of corticosterone, the predominant GC in rodents, were higher and the ERRFI1 gene expression was upregulated, whether $\beta_{2}$-AR is upregulated or blocked. Therefore, a reduction of Akt phosphorylation is expected. Accordingly, a reduced Akt phosphorylation has been reported in the ventricles of rats submitted to the same stress protocol used here ${ }^{21}$.

The present data have thus suggested that two signaling pathways are the most affected by stress: the $\beta$-ARGs-AC-cAMP and the PI3K-Akt signaling pathways. Moreover, the modulation of several of their components depends on the presence of the $\beta_{2}$-AR. The changes induced by stress in the $\beta$-AR-Gs-AC, GC-GR, and PI3K-Akt signaling pathways are summarized in Fig. 4. On the left side are listed some of the genes with regulation altered by stress (upregulation of ERRFI1, CREM, RGS1, HSP90aa1; and downregulation of APLN) and some predicted upstream molecules. The right side of Fig. 4 shows the genes that are altered and predicted upstream molecules when stress is applied in conjunction with the $\beta_{2}$-AR blockade: ERRFI1, CREM, HSP90aa1, and HSPA1A/A1B are upregulated whereas APLNR is downregulated.

The changes post stress in cardiac gene regulation also include the progression of the cell cycle, which can be impaired by downregulation of CDK1, and the presence of epigenetic factors, such as the upregulation of the class I histone deacetylase, HDAC8, and, in ICI-treated rats, stress induced upregulation of HSP40, and IRS2. The upregulation of IRS2 suggests that insulin may be an upstream regulator of the stress response, independent of $\beta_{2}-\mathrm{AR}$, since it appears in the comparisons of both non-ICI treated and ICI treated rats.

Effect of stress on components of the immune system. An unexpected finding was the recruitment of the immune system to the cardiac tissue of stressed rats, with the profile clearly different for the two groups, as a function of $\beta_{2}$-AR. Immunological activation was identified in microarray data in expressed genes, canonical pathways and upstream regulators. The correlation of immunological response with cardiac injury and repair is widely recognized in the failing heart ${ }^{46-48}$ and in human coronary heart disease ${ }^{49-51}$.

Among the differently expressed genes in the comparison of non-ICI treated group, IL22RA2 (interleukin 22 receptor subunit alpha 2), IL2RA (interleukin 2 receptor antagonist) and IFRD1 (interferon related developmental regulator 1) were upregulated and are described as regulators of the inflammatory response ${ }^{52,53}$. IL- 6 was also upregulated in this group. This is a pro-inflammatory cytokine produced by myeloid cells and cardiomyocytes in an autocrine mechanism ${ }^{54}$, which has been related to cardiac disease and the impairment of cardiac performance ${ }^{50,51}$.

It has been predicted that the pro-inflammatory cytokines IL-1 $\beta$ and TNF act as upstream regulators of the cardiac stress response in the presence of $\beta_{2}-A R$. Both are related to acute inflammation in cardiac disease, such as heart failure and myocardial infarction ${ }^{46,54}$. IL-1 $\beta$, TNF- $\alpha$, and IL-12 are cytokines released by T helper 1 lymphocytes (Th1), which control the cellular immune response. Interestingly, immune cells, including Th1 lymphocytes, express $\beta_{2}$-AR and are, therefore, susceptible to catecholamines action ${ }^{55}$. In immune cells, cAMPPKA signaling inhibits the transcription of nuclear factor kappa B (NF- $\kappa B$ ); through CREB, it activates the transcription of IL-10. This mechanism promotes the differentiation of the Th2 response, which is markedly antiinflammatory, and also inhibits the development of the Th1 response, which is markedly pro-inflammatory ${ }^{55,56}$. The chronic use of $\beta_{2}$-AR-selective and nonselective blockers in mice impairs the recruitment of leukocytes to the injured heart and reduces survival ${ }^{57}$. 
The data reported here thus suggest that in the presence of greater expression of $\beta_{2}$-AR, as previously reported by Moura et al. ${ }^{13}$, a pro-inflammatory signaling is triggered in the heart of stressed rats. If the upregulation of $\beta_{2}$-AR occurs only in the cardiomyocytes or in the resident immune cells as well is, however, unknown at the present time. Indeed, data for the comparison of stressed ICI-treated rats with non-stressed ICI-treated rats did not involve pro-inflammatory genes, which suggests that the treatment with ICI188,551 reduced the development of the Th1 response by blocking $\beta_{2}-A R$ in the immune cells as well. Several canonical pathways listed in that analysis are related to the immune response, such as the signaling pathway of IL-10, IL-17, CD40, CD27 and IL-6, the acute phase response, and GR signaling pathway. One interesting characteristic of these canonical pathways is the expression of the same gene, NFKBIA, at times in conjunction with MAP2K3 and/or JUN. NFKBIA encodes a potent inhibitor of NF- $\mathrm{KB}^{58}$, which mediates activation of the inflammatory response with important consequences in heart disease. The overexpression of NFKBIA in cardiomyocytes inhibits NF- $\kappa \mathrm{B}$ activity, reduces hypertrophy and improves cardiac performance and survival via the Akt signaling pathway ${ }^{59}$. The presence of NFKBIA in the left ventricle suppresses the expression of NF- $\kappa B$ and attenuates myocardial fibrosis ${ }^{60}$. Therefore, the expression of NFKBIA mRNA in ICI-treated stressed rats could indicate the presence of a regulatory or anti-inflammatory mechanism in the ventricle of rats submitted to stress when faced with $\beta_{2}$-AR blockage.

Study limitations and conclusion. The data has shown that stress induced alterations in the expression of such a large number of genes what seems to be part of adaptive mechanisms. $\beta_{2}$-AR clearly plays a role in this process, since the alterations in gene expression that occurred in the presence of this adrenoceptor subtype are completely different from those seen when it is blocked. However, it might be considered that there are many processes between the gene expression and the respective protein synthesis and activity. Indeed, one of the major challenges in all experimental systems is the issue of causality and association. Thus, in order to confirm if the altered gene expression here demonstrated has functional consequences, additional experiments are required. The lack of functional experiments and protein validation is a limitation of this work. Despite that, the great number of significant data presented here suggests that might be an influence of stress in the cardiac cells phenotype, and probably in the heart function.

\section{Methods}

Animals and experimental groups. Male Wistar rats (Rattus norvegicus; 24 animals; 250-350 g; 12 weeks-old), obtained from the Center for the Development of Experimental Models (CEDEME), of the Federal University of São Paulo (São Paulo, SP, Brazil), were housed in standard cages in a temperature-controlled room $\left(22^{\circ} \mathrm{C}\right)$ on a 12:12 h light:dark cycle, with the lights on at 7:00 am. Standard laboratory chow and tap water were available ad libitum. The rats were randomly distributed in two groups as follows: untreated and treated with ICI118,551 (ICI), with two subgroups each: submitted to stress and not submitted to stress. The experimental protocols were approved by the Ethics Committee for Animal Use of the Federal University of São Paulo (CEUA/UNIFESP), protocol number 86613101116, in accordance with the Brazilian National Council for Control of Animal Experimentation (CONCEA, Brazil), and all study carried out in compliance with the ARRIVE guidelines.

Stress protocol. The foot shock stress protocol was administered as previously described ${ }^{13,61,62}$. The rats in the stressed groups (both untreated and ICI-treated; 6 rats/group) were submitted to foot shock sessions; rats in the non-stressed groups (untreated and ICI-treated; 6 rats/group) were also placed in the foot shock cage, but did not receive foot shocks. The stress cage was a Plexiglas chamber $(26 \times 21 \times 26 \mathrm{~cm})$ provided with a floor grid made of stainless-steel rods $(0.3 \mathrm{~cm}$ in diameter, spaced $1.5 \mathrm{~cm}$ apart). During the daily 30 min stress sessions, which occurred between 7:30 am and 11:00 am on three consecutive days, foot shocks were delivered by a constant current source controlled by a microprocessor-based instrument. The intensity of the current was $1.0 \mathrm{~mA}$, with duration of $1.0 \mathrm{~s}$, with pulses delivered at random intervals of between 5 and $25 \mathrm{~s}$. The rats were returned to their standard cages after the first and second period in the Plexigas chamber. After the third session, the rats were immediately euthanized by decapitation, in compliance with the American Veterinary Medical Association (AVMA) Guidelines for the Euthanasia of Animals (2020). The trunk blood was collected in a tube containing EDTA and centrifuged. The plasma was separated and stored at $-80^{\circ} \mathrm{C}$. The hearts were harvested and the left ventricles were isolated and stored at $-80^{\circ} \mathrm{C}$.

Treatment with ICI118,551. For 5 days, the rats in the ICI-treated groups, stressed or not, received $500 \mu \mathrm{g} / \mathrm{kg} /$ day, i.p, of ICI118,551 (( \pm )-1-[2,3-(dihidro-7-metil-1H-inden-4-il) oxi]-3-[1-metilletil) amino]2-butanol; Tocris Bioscience, Bristol, UK), a highly selective $\beta_{2}-A R$ antagonist ${ }^{63}$. The rats in the untreated nonstressed control group and those submitted to stress that were not treated with ICI received injections of saline solution, i.p. The foot shock stress sessions began on the third day of the treatment with ICI or saline solution.

Plasma corticosterone concentration. The plasmatic concentration of corticosterone was determined in all experimental groups (6 rats/group) by enzyme immunoassay (ELISA) using a commercial kit (Enzo Life Science, Inc., Ann Arbor, MI, EUA) according to the manufacturer's guidelines.

RNA preparation and processing. Total RNA was extracted from fragments of up to $100 \mathrm{mg}$ from the left ventricle from all experimental groups (4 rats/group) using TRIzol reagent according to the manufacturer's instructions (Invitrogen, Carlsbad, CA, USA). The final volume of the samples was $100 \mu \mathrm{L}$ of water treated with $0.1 \%$ dietilpyrocarbonate (DEPC UltraPure; Invitrogen, Carlsbad, CA, USA); these were stored at $-80^{\circ} \mathrm{C}$ over- 
night. The mRNA concentration and degree of purity were determined in Nanodrop $2000 \mathrm{c}$ (Thermo Scientific, Waltham, MA, USA) under 260/280 nm. The RNA was purified using an RNeasy Kit (Qiagen, Valencia, CA, USA) according to the manufacturer's guidelines. The purified RNA concentration and degree of purity were determined in Nanodrop $2000 \mathrm{c}$.

Microarray assay. The gene expression profile was evaluated for the total RNA isolated from the left ventricles of the rats in each of the experimental groups. The two-color microarray-based gene expression analysis was Agilent-074036 SurePrint G3 Rat GE v2 8×60 K Microarray G4858A (GEO GPL22145) from Agilent Technologies (Santa Clara, CA, USA). There were a total of four samples for each group. Each sample was analyzed once. The analysis was performed at the Center of Excellence-Genomics (Agilent Technologies Brasil, Alphaville, Barueri, São Paulo, Brazil).

Firstly, the total RNA samples were assayed using RNA ScreenTape Analysis in a TapeStation System (Agilent Technologies, Santa Clara, CA, USA) to determine RNA quality and concentration (ng/ $\mu \mathrm{L}) .200 \mathrm{ng}$ of total RNA were used for individual reactions using oligo-dt linked to T7 promoter primer, being the Spike A Mix/Cyanine 3-CTP dye for the non-stressed samples and Spike B Mix/Cyanine 5-CTP dye for the stressed samples. The transcription of cDNA was performed using cDNA Master Mix for $2 \mathrm{~h}$ at $40^{\circ} \mathrm{C}$, followed by $15 \mathrm{~min}$ at $70^{\circ} \mathrm{C}$. The cDNA served as a template for the next transcription reaction. For the in vitro transcription of cRNA, Cyanine 3-CTP (for the non-stressed samples) and Cyanine 5-CTP (for the stressed samples) were incorporated, using T7 RNA polymerase for $2 \mathrm{~h}$ at $40^{\circ} \mathrm{C}$. After this, the samples were stored at $-80^{\circ} \mathrm{C}$. On the following day, the amplified and labeled cRNA was purified using an RNeasy kit, according to the manufacturer's guidelines, and the concentration was determined by RNA ScreenTape Analysis in a TapeStation System. For each microarray, $300 \mathrm{ng}$ portions of each labeled cRNA sample were assembled in pairs of Cyanine 3-CTP (for non-stressed samples) and Cyanine 5-CTP (for stressed samples) incorporation as follows: samples of non-stressed untreated with stressed untreated groups, and samples of non-stressed ICI treated with stressed ICI treated groups. Then they were fragmented for $30 \mathrm{~min}$ at $60^{\circ} \mathrm{C}$. The hybridization assembly was prepared according to the Agilent microarray hybridization chamber user guide. The hybridization was performed in the Agilent Microarray Hybridization Quick Assemble Chamber for $17 \mathrm{~h}$ at $65^{\circ} \mathrm{C}$ at a rotation speed of $10 \mathrm{rpm}$. The microarray slide was washed with gene expression wash buffers provided by Agilent Technologies, followed by an acetonitrile wash to avoid sediment and dry the slide. Agilent SureScan was used to scan the microarray slide.

Microarray data analysis. Data were extracted using Agilent Feature Extraction software. The fluorescence intensity for each spot was corrected by subtracting the background using the standard Feature Extraction algorithm. Then, data was normalized using Lowess algorithm, which is applied for two-color data to compensate dye bias incorporation. The metrics parameters were considered adequate. GeneSpring GX software (Agilent Technologies, Santa Clara, CA, USA) was used for differential gene expression analysis. Significant changes were defined using t test against zero on the basis of fold change $(\geq 2)$ and $p$ value $(\leq 0.05)$ cut offs. The log ratio (log two fold change) was taken. The functional significance of the genes with differential expression was determined in Ingenuity Pathway Analysis software (IPA; Qiagen, Valencia, CA, USA) by right-tailed Fisher's exact test with $\mathrm{p}$ value of overlap $\leq 0.05$. The $\mathrm{z}$ score $(\geq 2$ or $\leq-2)$ was considered.

Received: 5 January 2021; Accepted: 17 February 2021

Published online: 28 April 2021

\section{References}

1. Woo, A. Y. H. \& Xiao, R. P. $\beta$-Adrenergic receptor subtype signaling in heart: From bench to bedside. Acta Pharmacol. Sin. 33, 335-341 (2012).

2. Minneman, K. P., Hegstrand, L. R. \& Molinoff, P. B. Simultaneous determination of beta-1 and beta-2-adrenergic receptors in tissues containing both receptor subtypes. Mol. Pharmacol. 16, 34-46 (1979).

3. Juberg, E. N., Minneman, K. P. \& Abel, P. W. Beta 1- and beta 2-adrenoceptor binding and functional response in right and left atria of rat heart. Naunyn Schmiedebergs Arch. Pharmacol. 330, 193-202 (1985).

4. Brodde, O. E. Beta 1-and beta 2 -adrenoceptors in the human heart: Properties, function, and alterations in chronic heart failure. Pharmacol. Rev. 43, 203-242 (1991).

5. Spadari, R. C. et al. Role of beta-adrenergic receptors and sirtuin signaling in the heart during aging, heart failure, and adaptation to stress. Cell Mol. Neurobiol. 38, 109-120 (2018).

6. Callia, M. L. \& de Moraes, S. Heterogeneity of beta adrenoceptors in right atria isolated from cold-exposed rats. J. Pharmacol. Exp. Ther. 230, 450-454 (1984).

7. Bassani, R. A. \& de Moraes, S. Effects of repeated footshock stress on the chronotropic responsiveness of the isolated pacemaker of the rat: Role of beta-2 adrenoceptors. J. Pharmacol. Exp. Ther. 246, 316-321 (1988).

8. Esch, T., Stefano, G. B., Fricchione, G. L. \& Benson, H. Stress in cardiovascular diseases. Med. Sci. Monit. 8, RA93-RA101 (2002).

9. Santos, I. N., Marcondes, F. K. \& Spadari-Bratfisch, R. C. The beta1-adrenoceptor site activated by CGP 12177 varies in behavior according to the estrous cycle phase and stress. Can. J. Physiol. Pharmacol. 81, 459-468 (2003).

10. Santos, I. N. \& Spadari-Bratfisch, R. C. Stress and cardiac beta adrenoceptors. Stress 9, 69-84 (2006).

11. Wittstein, I. S. Acute stress cardiomyopathy. Curr. Heart Fail. Rep. 5, 61-68 (2008).

12. Steptoe, A. \& Kivimaki, M. Stress and cardiovascular disease. Nat. Rev. Cardiol. 9, 360-370 (2012).

13. Moura, A. L., Hyslop, S., Grassi-Kassisse, D. M. \& Spadari, R. C. Functional $\beta_{2}$-adrenoceptors in rat left atria: Effect of foot-shock stress. Can. J. Physiol. Pharmacol. 95, 999-1008 (2017).

14. Theorell, T. et al. Stress and cardiovascular disease. Eur. Heart Netw. 9, 360-370 (2006).

15. Vanderlei, L. C., Marcondes, F. K., Lanza, L. L. \& Spadari-Bratfisch, R. C. Influence of the estrous cycle on the sensitivity to catecholamines in right atria from rats submitted to foot shock stress. Can. J. Physiol. Pharmacol. 74, 670-678 (1996). 
16. Spadari-Bratfisch, R. C. \& dos Santos, I. N. Adrenoceptors and adaptive mechanisms in the heart during stress. Ann. N. Y. Acad. Sci. 1148, 377-383 (2008).

17. Bristow, M. R. et al. Reduced $\beta_{1}$ receptor messenger RNA abundance in the failing human heart. J. Clin. Invest. 92, 2737-2745 (1993).

18. Rudomanova, V. \& Blaxall, B. C. Targeting GPCR-G $\beta \gamma$-GRK2 signaling as a novel strategy for treating cardiorenal pathologies. Biochim. Biophys. Acta Mol. Basis Dis. 1863, 1883-1892 (2017).

19. Zhu, W. Z. et al. Dual modulation of cell survival and cell death by $\beta_{2}$-adrenergic signaling in adult mouse cardiac myocytes. Proc. Natl. Acad. Sci. 98, 1607-1612 (2001).

20. Zhang, W. et al. $\beta$-Adrenergic receptor-PI3K signaling crosstalk in mouse heart: Elucidation of immediate downstream signaling cascades. PLoS One 6, e26581. https://doi.org/10.1371/journal.pone.0026581 (2011).

21. Cordeiro, M. A., Rodrigues, L. S., Ortolani, D., de Carvalho, A. E. T. \& Spadari, R. C. Persistent effects of subchronic stress on components of ubiquitin-proteasome system in the heart. J. Clin. Exp. Cardiolog. 11, 676 (2020).

22. Greco, C. M. \& Condorelli, G. Epigenetic modifications and noncoding RNAs in cardiac hypertrophy and failure. Nat. Rev. Cardiol. 12, 488-497 (2015).

23. Müller, F. U., Neumann, J. \& Schmitz, W. Transcriptional regulation by cAMP in the heart. Mol. Cell Biochem. 212, 11-17 (2000).

24. Müller, F. U. et al. Impaired cardiac contraction and relaxation and decreased expression of sarcoplasmic $\mathrm{Ca}^{2+}$-ATPase in mice lacking the CREM gene. FASEB J. 17, 103-105 (2003).

25. Lewin, G. et al. Critical role of transcription factor cyclic AMP response element modulator in $\beta_{1}$-adrenoceptor-mediated cardiac dysfunction. Circulation 119, 79-88 (2009).

26. Mittmann, C. et al. Expression of RGS proteins in human myocardium: Functional characterization of an upregulation of RGS4 in heart failure. Cardiovasc. Res. 55(4), 778-786 (2002).

27. Tran, T. et al. Interferon $\beta-1 \mathrm{~b}$ induces the expression of RGS1 a negative regulator of G-protein signaling. Int. J. Cell. Biol. 2010, 529376. https://doi.org/10.1155/2010/529376 (2010).

28. Hu, X. et al. RGS1 silencing inhibits the inflammatory response and angiogenesis in rheumatoid arthritis rats through the inactivation of Toll-like receptor signaling pathway. J. Cell Physiol. 234, 20432-20442 (2019).

29. Lindsey, S. H., Liu, L. \& Chappell, M. C. Vasodilation by GPER in mesenteric arteries involves both endothelial nitric oxide and smooth muscle cAMP signaling. Steroids 81, 99-102 (2014).

30. Yu, X., Li, F., Klussmann, E., Stallone, J. N. \& Han, G. G protein-coupled estrogen receptor 1 mediates relaxation of coronary arteries via cAMP/PKA-dependent activation of MLCP. Am. J. Physiol. Endocrinol. Metab. 307, E398-407. https://doi.org/10.1152/ajpen do.00534.2013 (2014).

31. Pei, H. et al. G Protein-coupled estrogen receptor 1 inhibits angiotensin II-induced cardiomyocyte hypertrophy via the regulation of PI3K-Akt-mTOR signalling and autophagy. Int. J. Biol. Sci. 15, 81-92 (2019).

32. Kuba, K., Sato, T., Imai, Y. \& Yamaguchi, T. Apelin and Elabela/Toddler; double ligands for APJ/Apelin receptor in heart development, physiology, and pathology. Peptides 111, 62-70 (2019).

33. Mughal, A. \& O'Rourke, S. T. Vascular effects of apelin: Mechanisms and therapeutic potential. Pharmacol. Ther. 190, 139-147 (2018).

34. Ashley, E. A. et al. The endogenous peptide apelin potently improves cardiac contractility and reduces cardiac loading in vivo. Cardiovasc. Res. 65, 73-82 (2005).

35. Chandrasekaran, B., Dar, O. \& McDonagh, T. The role of apelin in cardiovascular function and heart failure. Eur. J. Heart Fail. 10, 725-732 (2008).

36. Chandrasekaran, B. et al. Myocardial apelin production is reduced in humans with left ventricular systolic dysfunction. J. Cardiac Failure 16, 556-561 (2010).

37. Parikh, V. N. et al. Apelin and APJ orchestrate complex tissue-specific control of cardiomyocyte hypertrophy and contractility in the hypertrophy-heart failure transition. Am. J. Physiol. Heart Circ. Physiol. 315, H348-H356 (2018).

38. Bers, D. M. Calcium cycling and signaling in cardiac myocytes. Annu. Rev. Physiol. 70, 23-49 (2008).

39. Ranek, M. J., Stachowski, M. J., Kirk, J. A. \& Willis, M. S. The role of heat shock proteins and co-chaperones in heart failure. Philos. Trans. R. Soc. 373, 20160530. https://doi.org/10.1098/rstb.2016.0530 (2018).

40. Vandevyver, S., Dejager, L. \& Libert, C. Comprehensive overview of the structure and regulation of the glucocorticoid receptor. Endocr. Rev. 35, 671-693 (2014).

41. Liu, B., Zhang, T. N., Knight, J. K. \& Goodwin, J. E. The glucocorticoid receptor in cardiovascular health and disease. Cells 8, 1227. https://doi.org/10.3390/cells8101227 (2019).

42. Tarone, G. \& Brancaccio, M. Keep your heart in shape: Molecular chaperone networks for treating heart disease. Cardiovasc. Res. 102, 346-361 (2014).

43. Zhu, W. S. et al. Hsp90aal: A novel target gene of miR-1 in cardiac ischemia/reperfusion injury. Sci. Rep. 6, 24498. https://doi.org/ 10.1038/srep24498 (2016).

44. Xu, D., Makkinje, A. \& Kyriakis, J. M. Gene 33 is an endogenous inhibitor of ppidermal growth factor (EGF) receptor signaling and mediates dexamethasone-induced suppression of EGF function. J. Biol. Chem. 280, 2924-2933 (2005).

45. Cai, J. et al. Targeted expression of receptor-associated late transducer inhibits maladaptive hypertrophy via blocking epidermal growth factor receptor signaling. Hypertension 53, 539-548 (2009).

46. Zhang, Y., Bauersachs, J. \& Langer, H. F. Immune mechanisms in heart failure. Eur. J. Heart Fail. 19, 1379-1389 (2017).

47. Plenz, G. et al. Activation of the cardiac interleukin-6 system in advanced heart failure. Eur. J. Heart Fail. 3, 415-421 (2001).

48. von Haehling, S., Schefold, J. C., Lainscak, M., Doehner, W. \& Anker, S. D. Inflammatory biomarkers in heart failure revisited: Much more than innocent bystanders. Heart Fail. Clin. 5, 549-560 (2009).

49. Li, H. et al. Inflammatory biomarkers of coronary heart disease. Front. Biosci. 10, 185-196 (2018).

50. Swerdlow, D. I. et al. The interleukin-6 receptor as a target for prevention of coronary heart disease: A mendelian randomisation analysis Interleukin-6 Receptor Mendelian Randomisation Analysis (IL6R MR). Lancet 379(9822), 1214-1224 (2012).

51. Sarwar, N. et al. Interleukin-6 receptor pathways in coronary heart disease: A collaborative meta-analysis of 82 studies IL6R Genetics Consortium Emerging Risk Factors Collaboration. Lancet 379(9822), 1205-1213 (2012).

52. Micheli, L. et al. PC4/Tis7/IFRD1 Stimulates skeletal muscle regeneration and is involved in myoblast differentiation as a regulator of MyoD and NF-kB. J. Biol. Chem. 286, 5691-5707 (2011).

53. Trevejo-Nunez, G. et al. Interleukin-22 (IL-22) binding protein constrains IL-22 activity, host defense, and oxidative phosphorylation genes during pneumococcal pneumonia. Infect. Immunol. 87, e00550-e619. https://doi.org/10.1128/IAI.00550-19 (2019).

54. Epelman, S., Liu, P. P. \& Mann, D. L. Role of innate and adaptive immune mechanisms in cardiac injury and repair. Nat. Rev. Immunol. 15, 117-129 (2015).

55. Elenkov, I. J., Wilder, R. L., Chrousos, G. P. \& Vizi, E. S. The sympathetic nerve-an integrative interface between two supersystems: The brain and the immune system. Pharmacol. Rev. 52, 595-638 (2000).

56. Ağaç, D., Estrada, L. D., Maples, R., Hooper, L. V. \& Farrar, J. D. The $\beta_{2}$-adrenergic receptor controls inflammation by driving rapid IL-10 secretion. Brain Behav. Immun. 74, 176-185 (2018).

57. Grisanti, L. A. et al. Prior beta blocker treatment decreases leukocyte responsiveness to injury. JCI Insight. 4, e99485. https://doi. org/10.1172/jci.insight.99485 (2019). 
58. Hoffmann, A., Levchenko, A., Scott, M. L. \& Baltimore, D. The IkappaB-NF-kappaB signaling module: Temporal control and selective gene activation. Science 298, 1241-1245 (2002).

59. Higuchi, Y. et al. Cardioprotection afforded by NF-kB ablation is associated with activation of Akt in mice overexpressing TNF- $\alpha$. Am. J. Physiol. Heart Circ. Physiol. 290, H590-H598 (2006).

60. Ogata, T. et al. Myocardial fibrosis and diastolic dysfunction in deoxycorticosterone acetate-salt hypertensive rats is ameliorated by the peroxisome proliferator-activated receptor-alpha activator fenofibrate, partly by suppressing inflammatory responses associated with the nuclear factor-kappa-B pathway. J. Am. Coll. Cardiol. 43, 1481-1488 (2004).

61. Santos, I. N. \& Spadari-Bratfisch, R. C. Chronotropic response to (+/-)-CGP12177 in right atria of stressed rats. Can. J Physiol. Pharmacol. 79, 393-399 (2001).

62. Ortolani, D., Oyama, L. M., Ferrari, E. M., Melo, L. L. \& Spadari-Bratfisch, R. C. Effects of comfort food on food intake, anxietylike behavior and the stress response in rats. Physiol. Behav. 103, 487-492 (2011).

63. Ruffolo, R. R. J. In Adrenoceptors: Structure, Function and Pharmacology (ed. Ruffolo, R. R. J.) 279 (Hardwood Academic Luxembourg, 1995).

\title{
Acknowledgements
}

This work was supported by the São Paulo Research Foundation (FAPESP Grants 2016/20777-8, 2016/20784-4), Coordenação de Aperfeiçoamento de Pessoal de Nível Superior (CAPES), and Conselho Nacional de Desenvolvimento Científico e Tecnológico (CNPq Grant 424114/2016-0). The authors wish to thank Agilent Technologies Brasil (Alphaville, Barueri, São Paulo, Brazil), Dr. Flavia Pidone for technical assistance, and Linda El'Dash for the language edition.

\section{Author contributions}

A.E.T.S.C. designed the research, performed the experiments, analyzed data and prepared figures; M.A.C., and L.S.R. analyzed data and contributed to the preparation of the manuscript; D.O. performed the experiments and contributed to the preparation of the manuscript; R.C.S. designed the research, and supervised the experiments and data analysis; A.E.T.S.C. and R.C.S. prepared the manuscript. All authors reviewed the manuscript.

\section{Competing interests}

The authors declare no competing interests.

Additional information

Supplementary Information The online version contains supplementary material available at https://doi.org/ 10.1038/s41598-021-88267-8.

Correspondence and requests for materials should be addressed to A.E.T.S.d.C. or R.C.S.

Reprints and permissions information is available at www.nature.com/reprints.

Publisher's note Springer Nature remains neutral with regard to jurisdictional claims in published maps and institutional affiliations.

\begin{abstract}
Open Access This article is licensed under a Creative Commons Attribution 4.0 International License, which permits use, sharing, adaptation, distribution and reproduction in any medium or format, as long as you give appropriate credit to the original author(s) and the source, provide a link to the Creative Commons licence, and indicate if changes were made. The images or other third party material in this article are included in the article's Creative Commons licence, unless indicated otherwise in a credit line to the material. If material is not included in the article's Creative Commons licence and your intended use is not permitted by statutory regulation or exceeds the permitted use, you will need to obtain permission directly from the copyright holder. To view a copy of this licence, visit http://creativecommons.org/licenses/by/4.0/.
\end{abstract}

(c) The Author(s) 2021 